\title{
Liver glycogen in type 2 diabetic mice is randomly branched as enlarged aggregates with blunted glucose release
}

\author{
Quinn Alexander Besford ${ }^{\dagger}$. Xiao-Yi \\ Zeng $^{\ddagger} \cdot$ Ji-Ming Ye Y $^{\ddagger}$ Angus Gray-Weale ${ }^{\dagger}$
}

Received: date / Accepted: date

\begin{abstract}
Glycogen is a vital highly branched polymer of glucose that is essential for blood glucose homeostasis. In this article, the structure of liver glycogen from mice is investigated with respect to size distributions, degradation kinetics, and branching structure, complemented by a comparison of normal and diabetic liver glycogen. This is done to screen for differences that may result from disease. Glycogen $\alpha$-particle (diameter $\sim 150 \mathrm{~nm}$ ) and $\beta$-particle (diameter $\sim 25 \mathrm{~nm}$ ) size distributions are reported, along with in vitro $\gamma$-amylase degradation experiments, and a small angle X-ray scattering analysis of mouse $\beta$-particles. Type 2 diabetic liver glycogen upon extraction was found to be present as large loosely bound, aggregates, not present in normal livers. Liver glycogen was found to aggregate in vitro over a period of 20 hours, and particle size is shown to be related to rate of glucose release, allowing a structure-function relationship to be inferred for the tissue specific distribution of particle types. Application of branching theories to small angle X-ray scattering data for mouse $\beta$-particles revealed these particles to be randomly branched polymers, not fractal polymers. Together, this article shows that type 2 diabetic liver glycogen is present as large aggregates in mice, which may contribute to the inflexibility of interconversion between glucose and glycogen in type 2 diabetes, and further that glycogen particles are randomly branched with a size that is related to the rate of glucose release.
\end{abstract}

Keywords Diabetes · Glycogen · Dynamic Light Scattering · Small Angle X-ray Scattering · Fractal · Randomly Branched

\footnotetext{
$\dagger$

School of Chemistry, University of Melbourne, Victoria 3010, Australia

E-mail: quinn.besford@gmail.com E-mail: angusg@unimelb.edu.au

School of Health Sciences, RMIT University, Victoria 3083, Australia E-mail: jiming.ye@rmit.edu.au
} 


\section{Introduction}

Glycogen is a highly branched polysaccharide present in most organisms. In mammals, glycogen is mainly present in liver, skeletal muscle, cardiac, brain, and adipose tissue [1]. The specific function of glycogen may differ for each tissue, but broadly has two main purposes: to act as a glycolytic fuel [2], and to anchor glucose out of the blood and maintain the cellular osmotic pressure [3]. The function of glycogen may be correlated to the structure present in a particular tissue, where glycogen mainly exists as two different forms. In the skeletal muscle of normal mice and humans [4, 1] glycogen exists as roughly spherical particles about $25 \mathrm{~nm}$ in diameter, called $\beta$-particles. In the liver and hearts of normal mice and rats $[5,6]$, $\beta$-particles are assembled, apparently covalently [7], into larger $\alpha$-particles about $150 \mathrm{~nm}$ in diameter. Both liver and cardiac glycogen is heterogeneous as it consists of both dispersed $\beta$-particles and larger $\alpha$-particles of varying size. The size distributions of the larger $\alpha$-particles varies considerably between tissues, organisms, and even life forms $[8,9]$. This variation between tissues and organisms suggests a structure-function relationship.

Methods for the study of glycogen structure include enzyme and chemical degradation experiments $[10,11]$, size exclusion chromatography (SEC) with inline detection that provide number and weight distributions [12], transmission electron microscopy (TEM) that allows visualisation of the particles [1], and dynamic light scattering (DLS) that provides hydrodynamic particle size distributions [6]. These experiments have revealed much information about glycogen, but questions still remain regarding the distribution of mass within the structure and how the mass is arranged; what is the branching structure? This structural information will allow glycogen to be better understood with respect to a structure-function relationship.

The structure of glycogen $\beta$-particles has been described as highly optimised and fractal [13, 14, 15]. Melendez-Hevia et al. [14] suggested that glycogen is made of successive regular tiers, based on a model of optimised parameters for branching and chain lengths across the molecule. An alternative model, called random branching theory (RBT), developed by Konkolewicz et al. [16, 17], has been used to study the distribution of mass with growth of hyperbranched polymers. According to RBT, the radius $r$ of a randomly hyperbranched polymer scales as log $M_{w}$, where $M_{w}$ is the molecular weight of the polymer. The ideal fractal, on the other hand, has a self-similar structure and possesses a mass distribution which is independent of size and obeys $r \propto M_{w}^{D_{f}^{-1}}$, where $D_{f}$ is the fractal dimension [18]. A direct test of these competing models is needed to elucidate the branching structure of glycogen $\beta$-particles.

Small angle X-ray scattering (SAXS) allows the distribution of electron density within a macromolecule to be directly probed and subsequently modelled [19]. Goldsmith et al. [20] determined the structure of maltoheptaose from X-ray scattering experiments, showing the short linear oligomer forms a helical structure. Filippov et al. [21] reported a variety of experiments on functionalised commercial oyster glycogen which included some SAXS, though the particles were found to be aggregated. Obtaining SAXS data for glycogen $\beta$-particles will allow the test of a structure theory, such as Konkolewicz et al.'s [22] RBT model. Konkolewicz et al. [22] successfully demonstrated the application of RBT to SAXS data of 
dendrimers, therefore such application on glycogen would provide a direct test of internal structure as to whether the branching is fractal or randomly branched. Establishing the structural nature of the $\beta$-particles will then allow a benchmark to be set to compare for differences in diseased, for example diabetic, organisms. Before comparing for differences in diseased organisms it is first important to understand the physiological role of glycogen in different tissues, and where structural differences may result in disease.

Glycogen is distributed throughout most mammalian tissues, some with more than others; about $10 \%$ of the weight of the liver is glycogen, compared to $2 \%$ for muscle [23]. There are clear differences in glycogen's purpose in different tissues. Liver and kidney tissues are the only tissues that release glucose into the blood in times during hypoglycaemia (low blood glucose) [24]. On the other hand, during periods of hyperglycaemia (high blood glucose) the secretion of insulin stimulates glucose uptake from the blood into mostly skeletal muscle and adipose tissue $[25,26,27]$, and at the same time glucose production in the liver is suppressed [28]. Therefore at least two differences in glycogen's function exist for these different tissues; the liver and kidneys function to release glucose into the blood from glycogen, and the skeletal muscle and adipose tissue keep glucose out of the blood by storing glucose as glycogen. The different size distributions of glycogen between these tissues ( $\alpha$-particles in liver and $\beta$-particles in skeletal muscle) hints at a relationship between glycogen size and its biological role.

It is well established that an inability to form normal glycogen particles, such as $\alpha$ and $\beta$-particles, results in the tissue's inability to function, such as in the glycogen storage diseases where glycogen is either too abundant or has an abnormal structure [29], or in Lafora disease which is thought to result from an over-phosphoylation of glycogen which changes the chain lengths and solubility of glycogen [30,31]. Other conditions that involve an inability to regulate blood glucose levels correctly may therefore have different glycogen structures or size distributions in comparison to normal. The main interest in this article is diabetic liver glycogen, and its role in hypoglycaemia. The main target of glucagon stimulated glucose release in periods of hypoglycaemia is the liver tissue [32], therefore the poor response to hypoglycaemia seen in type 2 diabetics (T2D) medicated with insulin [33, 34] may in part be due to a lack of normal liver glycogen replenishment postprandially or post-exercise [35], failure of the enzyme cascade leading to glycogen degradation (such as in some glycogen storage diseases [36]), or an alternate structure of the large polymer [37].

Since glycogen structure seems to be tissue specific, a specific function needs to be correlated to the size distributions of glycogen particles. A comparison of particle size, degradation kinetics, and internal structure may allow a greater examination of the structure-function relationship for glycogen particles, and ultimately lead towards new insight into how structural abnormalities could result, or be managed in disease. This article reports a rapid screening method to determine liver glycogen size distributions of $\alpha$ and $\beta$-particles across normal, type 1 diabetic (T1D) and type 2 diabetic (T2D) model mice, accompanied by a degradation kinetics analysis of differently sized glycogens. SAXS measurements of mammalian glycogen $\beta$-particles are reported along with applied branching theory, used to determine in greater detail the internal structure of glycogen $\beta$-particles. By combining SAXS, rapid DLS, and degradation kinetics experiments, new information 
on this vital biopolymer is found which allows a better understanding of its biological function.

\section{Methods}

2.1 Animals and glycogen purification

The animals and glycogen purification method are as reported by Zeng et al. [38] and Besford et al. [6], respectively. The fasting blood glucose of the animals sacrificed is given in Table 1 .

\subsection{Dynamic light scattering (DLS)}

DLS measurements were carried out on a Malvern Zetasizer Nano (ZS) instrument, fitted with a $4 \mathrm{~mW}$ He-Ne laser $(633 \mathrm{~nm})$. A standard operating procedure was used with a back-scattering detector position of $4.33 \mathrm{~mm}$. Back-scattering detection was used to give an increased sensitivity to the smaller $\beta$-particles [39]. The intensity size distributions were obtained from analysis of the correlation functions using the Multiple Narrow Modes algorithm in the dispersion technology software (DTS). Measurements were carried out in deionised water obtained using a Milli-Q system (Millipore). Probability distributions were fitted to the DLS intensity distributions in order to obtain clear statistics (see supporting information). DLS, although biased towards larger particles, reveals both $\alpha$ and $\beta$-particle distributions, where hydrolysis of $\alpha$-particles (see SAXS method) causes the $\beta$-particle distribution to increase substantially, as shown in Figure 1 . $\zeta$-potentials were measured on the same instrument.

\subsection{Glycogen degradation experiments}

Glycogen particles were incubated with a reaction mixture of $0.5 \mathrm{mg} / \mathrm{ml}$-aminoantipyrene, $1.6 \mathrm{U} / \mathrm{ml}$ peroxidase, $10 \mathrm{U} / \mathrm{ml}$ glucose oxidase, $50 \mathrm{mU} / \mathrm{ml}$ amyloglucosidase $(\gamma$ amylase), $0.12 \mathrm{M} \mathrm{NaH}_{2} \mathrm{PO}_{4}, 1 \mathrm{mg} / \mathrm{ml}$ phenol, at $\mathrm{pH} 7.0$ and $37^{\circ} \mathrm{C}$. The absorbance was continuously measured at $490 \mathrm{~nm}$ for 90 minutes. The kinetics were fitted via

$$
A=A_{\infty}\left(1-e^{-t / \tau_{r}}\right),
$$

where $A$ is absorbance at time $t, A_{\infty}$ is the absorbance once the reaction is fully completed, and $\tau_{r}$ is the relaxation time of the enzyme or the reciprocal of the enzyme adsorption rate, expressed in reciprocal minutes. Aminoantipyrine was purchased from MP Biomedicals, Australia. All other chemicals were purchased from Sigma-Aldrich, Australia.

\subsection{Small angle X-ray scattering (SAXS)}

SAXS was carried out at the SAXS beamline of the Australian Synchrotron, using the $7 \mathrm{~m}$-camera setup detected with a $1 \mathrm{M}$ Pilatus detector. Glycogen $\alpha$-particles 
were hydrolysed to dispersed $\beta$-particles by treating the $\alpha$-particles with $1 \mathrm{M} \mathrm{HCl}$. Samples were sonicated and vortexed over a period of about $20 \mathrm{~min}$. Hydrolysis was confirmed via DLS, shown in Figure 1. A 4-step concentration series were made of each sample, and samples were then loaded into a 96-well plate, and centrifuged at 3000rpm for about 10 minutes.

\section{Results/Discussion}

\subsection{DLS particle size distributions of $\alpha$ and $\beta$-particles}

Results for DLS size distributions of liver glycogen from normal and diabetic mice are shown in Figure 2, where each distribution is the average of three experiments and the standard deviations are shown as error bars. For normal mice the average liver glycogen $\alpha$-particle peak position, across the 11 animals studied, is $147 \pm 25$ $\mathrm{nm}$ (see Figure $2 \mathrm{~A}$ ), consistent with data reported for rat liver glycogen particles of $158 \pm 48 \mathrm{~nm}$ [1], and with transmission electron microscopy (TEM) of mouse liver glycogen at $160 \pm 41 \mathrm{~nm}$ [6]. Very little variability between these normal glycogen distributions is found for both $\alpha$ and $\beta$-particle distributions, though DLS may not be sensitive enough to detect variability in the $\beta$-particle distributions. The animals used in this study were sacrificed at similar times in their diurnal cycle, therefore consistent distributions seen in Figure 2 A may reflect this.

The T1D particle size distributions, shown in Figure 2 B, have very similar size distributions to normal liver glycogen with the $\alpha$-particles consistent at a position of $143 \pm 2 \mathrm{~nm}$. The T2D glycogen, on the other hand, shown in Figure $2 \mathrm{C}$, has substantial variability across the 20 mice studied. T2D glycogen is seen to consist of larger size distributions than both normal and T1D liver glycogen (note the log scale). These distributions are not only larger, but are also variable in size with large standard deviations; some of the T2D particle size distributions reach above $400 \mathrm{~nm}$. These larger particle sizes may affect the enzymatic degradation kinetics of these glycogens, especially if size is related to function (see kinetics analysis below). The large standard deviations from the mean of these T2D distributions may indicate irregular scattering from the larger particles, but may also hint at an increased lability of the particles, as in unstable aggregates. To determine the nature of these larger particles, whether covalent or labile aggregates, the T2D glycogen samples were subjected to intense sonication and re-analysed via DLS with the particle size distributions shown in Figure 2 D.

Figure $2 \mathrm{D}$ shows that upon intense sonication the T2D particle size distributions revert to distributions resembling those found in normal mice (Figure 2 A). The particle distributions across the T2D post sonication are very consistent, where the $z$-average diameter, which is a cumulant mean from a polynomial fit to the first order autocorrelation function, for the original T2D samples was $269 \pm$ $138 \mathrm{~nm}$, post sonication is $106 \pm 33 \mathrm{~nm}$, remarkably close to normal glycogen at $107 \pm 12 \mathrm{~nm}$. Note that these diameters are an average over the $\alpha$ and $\beta$-particle distributions, whereas the previous diameters are for $\alpha$-particles only. These results point to noncovalent aggregation of T2D glycogen particles, an aggregation not seen in normal or T1D liver glycogens.

To better compare the variability between the four sets of conditions, probability functions were fitted to the DLS data to allow integration up to the quartiles 
(details in supporting information), shown as a boxplot in Figure 3. It is clear from Figure 3 that T2D liver glycogen, upon extraction and characterisation, is in a much larger aggregate form, evidenced from the resulting dispersion after sonication. Figure 3 emphasises how close the particle size distributions between these normal, T1D, and sonicated T2D glycogens are. All the T2D glycogen samples were treated in an identical manner to the T1D and normal glycogens, showing that these aggregated structures are not an artefact of analysis. This analysis confirms that covalent $\alpha$-particles are present in T2D livers, and further supports the conclusion that T2D glycogen forms labile aggregates.

Other work by Sullivan et al. [37] involved screening liver glycogen particles from normal and genetically T2D mice via SEC with dimethyl sulfoxide (DMSO) and $\mathrm{LiBr}$ as the solvent and eluent. The authors found that T2D liver glycogen predominately consists of $\beta$-particles. Later, Deng et al. [40] found that when aqueous SEC is used (as opposed to DMSO based), T2D liver glycogen is present as $\alpha$-particles that resemble normal. This may indicate a different type of binding in the T2D $\alpha$-particles, as suggested by Deng et al. [40]. However, an important point is that Deng et al. subjected the particles to an 8 hour pre-treatment in a thermomixer at $80^{\circ} \mathrm{C}$, and subsequently observed T2D liver glycogen similar to normal. This result is therefore consistent with our results for T2D, where post sonication size distributions match normal (Figure 2). The advantage of DLS is the absence of sheer, which reveals as noted above the change in attractive forces for $\mathrm{T} 2 \mathrm{D}$, through detection of fragile aggregates.

The gentle particle sizing analyses performed here has shown that T2D liver glycogen is originally present as large labile aggregates, and when this is subjected to a sonication treatment size distributions resembling normal glycogen results. This shows that T2D liver glycogen does consist of $\alpha$-particles, in agreement with Deng et al. [40]. The nature of the original large aggregate T2D glycogens, along with possibly consequences for an organism with them, is discussed in the following sections. First, the cause behind glycogen aggregating is investigated with respect to $\zeta$-potentials.

\subsection{1 $\zeta$-potentials and aggregation of glycogen particles}

The degree of phosphorylation on the glycogen particles may affect the dispersion in solution. Low phosphorylation may result in less double-layer repulsion between the glycogen particles and the surrounding counterions, ultimately leading to aggregation, whereas high phosphorylation may result in greater repulsion and therefore dispersion of particles in solution. As an indication of this, the $\zeta$-potentials of the glycogens in deionised water were measured. This gives an indication of a particle's charge (the $\zeta$-potential is defined as the electrical potential between the inner Helmholtz layer near a particles surface and the bulk liquid in which it is suspended [41]). From this information one may infer the strength of electric double layer repulsion and therefore likelihood of flocculation between particles. The $\zeta$-potential of all glycogens in water are roughly the same at around $-20 \mathrm{mV}$ (see Table 2), which is consistent with a small amount of phosphate on the particles, in agreement with previous work [42]. These $\zeta$-potentials indicate that the particles will repel one another, but when given enough time, may aggregate.

If the aggregation of T2D glycogen is due to reduced phosphate (charge) one might expect a different $\zeta$-potential in comparison to normal. Given the $\zeta$ - 
potentials are about the same, the explanation for T2D aggregation must lay elsewhere. Since the BGLs in a T2D are generally higher than optimal, the liver glycogen may be underutilised. T2D's have been shown to have a reduced rate of glycogenolysis (breakdown of glycogen to yield glucose) than normal subjects [43], and also to have greater stores of glycogen after 3-day fasting than normal [44]. This could result in T2D liver glycogen being present for longer periods in vivo, possibly allowing enough time for aggregation to occur.

The aggregation behaviour of glycogen was investigated by continuous DLS measurements of normal glycogen particles under different conditions over a period of 20 hours. These included suspension in Milli-Q water, and in PBS buffer at $25^{\circ} \mathrm{C}$, and also in PBS buffer at biological temperature $\left(37^{\circ} \mathrm{C}\right)$. The $z$-average diameter, the average of all particles $(\alpha$ and $\beta$ ), for these systems is shown in Figure 4.

Figure 4 shows that normal glycogen, in all three systems studied, aggregates with time. This aggregation occurs within the first 5 hours of dispersion into solution, and continues over 20 hours until aggregate size becomes limited by concentration. Some differences are seen between the three systems, where normal glycogen in water at room temperature $\left(25^{\circ} \mathrm{C}\right)$ aggregates to a z-average diameter of about $300 \mathrm{~nm}$, whereas that for both PBS buffer systems aggregates to about $200 \mathrm{~nm}$. These diameters are similar to the z-average for T2D samples in Figure 2 $\mathrm{C}$ of $269 \pm 138 \mathrm{~nm}$. Most importantly, the PBS system that resembles both a biological solvent and temperature aggregates with time. The enlarged glycogen aggregates found in T2D livers may therefore result from underutilised glycogen stores that are present for long periods of time in vivo. Consequences of larger glycogen particles is investigated in the following section.

\subsection{Glycogen degradation kinetics}

Given the broad distribution of glycogen particle size between tissues and organisms (see introduction), the size of glycogen is likely to be related to its function, hence the T2D aggregate particles may have a consequence for glucose metabolism and storage. The aggregate particles will have a reduced surface accessible area in comparison to the dispersed $\alpha$ and $\beta$-particles. Enzyme access may therefore be restricted on these glycogens, potentially resulting in less available glucose when needed, such as in periods of hypoglycaemia. The rate of enzyme action on differently sized glycogens was quantified by coupling an in vitro degradation using $\gamma$-amylase to a simple glucose assay with absorbance being measured with time. The glycogens analysed are of $\beta$-particle type (oyster [8] and rabbit liver [1]), $\alpha$ and $\beta$-particles together (T1D), and aggregate $\alpha$ and $\beta$-particles together (T2D). The kinetics were fitted with a simple function (see methods) in order to obtain the relaxation time for the enzymatic degradation. The results are shown in Table 3.

Table 3 reveals that the smaller glycogen $\beta$-particles are degraded by $\gamma$ amylase at a much faster rate than the larger T1D and aggregated T2D glycogen particles. Furthermore, there are differences between the relaxation times between T1D $\alpha$-particles and T2D aggregate glycogens demonstrating that glucose release is blunted in the T2D particles due to the aggregated size. These results show that the size of glycogen particles is related to the rate of glucose release. This is a simple but biologically important structure-function relationship, because the size of glycogen particles varies between tissues, and now it is shown that different 
particle sizes release glucose at different rates. The distribution of $\beta$-particles in skeletal muscle tissues, and $\alpha$-particles in the liver and heart tissues, may now be understood with respect to this structure-function relationship; fast release ( $\beta$ particles) vs slow release ( $\alpha$-particles) of glucose in times of need. Release rate is likely proportional to the surface area of the particles, however, this cannot be directly confirmed due to the heterogeneity of glycogen $\alpha$-particles.

The altered glycogen particle size in T2D mouse livers may be responsible for, or contribute to, the poor response to hypoglycaemia in T2D [33]. Additionally, the abnormal size may result in the liver not being able to store as much glucose as glycogen. This finding therefore demonstrates a contribution to the inflexibility of interconversion between glucose and glycogen in type 2 diabetes. Now that this structural change in T2D has been identified, a new line of investigation into liver glycogen metabolism in T2D can follow. Aggregated glycogen particles are found in T2D livers (Figure $2 \mathrm{D}$ ), but perhaps they are elsewhere, such as in the heart muscle, which was recently shown to contain $\alpha$-particles in normal mice [6].

\subsection{Small angle X-ray scattering (SAXS) of $\beta$-particles}

Next, the internal structure of $\beta$-particles was investigated by small angle X-ray scattering (SAXS) and is discussed in this section.

Glycogen $\alpha$-particles are polydisperse, where the distribution of normal glycogen particle diameters can span over hundreds of nm's. There are also large $\alpha$ and $\beta$-particle aggregate glycogens, as seen in T2D livers in Figure 2 C. Since the scattering data obtained through SAXS corresponds to the average scattering over all particles in the ensemble, the glycogen particles used for SAXS analysis should be relatively monodisperse. For this to be achieved a gentle acid hydrolysis of the $\alpha$-particles into the smaller roughly spherical $\beta$-particles was performed (see methods and Figure 1). Hydrolysis was confirmed via DLS, where the $\alpha$-particles of diameter $\approx 150 \mathrm{~nm}$, reduced down to $\approx 44 \mathrm{~nm}$. Figure 1 shows a clear example of this occurring. SAXS was performed on these $\beta$-particles immediately after the hydrolysis treatment to avoid $\beta$-particles aggregating together. Linearity between scattering intensity when the momentum transfer $q$ is $0\left(I_{0}\right)$, and the concentration of the glycogen samples is shown in supporting information, done so to confirm no aggregation or association in solution.

The solvent-subtracted scattering curves for these normal, T1D, and T2D glycogen $\beta$-particles are shown in Figure 5, along with a scattering law which is described below. Each animal group is offset for clarity (see caption), and every scattering curve normalised with respect to $I_{0}$. Figure 5 shows little difference between normal, T1D, and T2D $\beta$-particles. The T2D $\beta$-particles, and to a lesser degree the T1D $\beta$-particles, hint at a small degree of aggregation, where the scattering intensity increases at low $q$. Whereas the normal samples show a more consistent plateau at low $q$. From a practical standpoint, the lowest $q$ part of the SAXS curve is dictated by a single size parameter [19]; the radius of gyration $r_{g}$. In the following this information is extracted and compared to DLS for independent confirmation of the $\beta$-particle size being analysed.

Extrapolation of the scattering data in the Guinier region for globular particles $\left(q r_{g}<1.3\right)$ allows an estimate of the scattering at $q=0, I_{0}$, to be obtained (see supporting information). The Guinier approximation was used to transform 
the low $q$ data into a $r_{g}$, and subsequently compared the SAXS estimate to the DLS result for the hydrodynamic radius of the hydrolysed $\alpha$-particles, in Table 4. Considering the standard errors, these radii from different experiments compare well with one another. It should be noted that they have different meanings; $r_{h}$ is derived from the particles diffusion coefficient using the Stokes-Einstein relation, whereas $r_{g}$ is the square root of the average squared distance of each scatterer from the particle's centre. This comparison gives independent confirmation that the SAXS profiles are of particles of about $\beta$-particle size. Table 4 also shows that T2D $\beta$-particles are slightly larger, on average, than both normal and T1D $\beta$-particles, but this difference is only marginal.

Figure 6 compares the pair-distance distributions of electrons in the glycogen $\beta$-particles, calculated through an indirect Fourier transformation of the scattering curves in Figure 5 [19]. The normal $\beta$-particles (see Figure 6 A) show a relatively consistent distribution of electron-electron distances, spiking at about $150 \AA$ and decaying steadily as the distances span the diameter of the particles. Similar distributions are seen with the T1D samples, but with a slightly extended decay to larger distances (Figure $6 \mathrm{~B}$ ). The T2D samples are noticeably less consistent in the distribution of distances, as seen in Figure 6 C. Since the T2D $P(r)$ functions span the differences of the normal and T1D $\beta$-particles, no direct conclusions about the distribution of density within these T2D samples can be established from these measurements, at least without better statistics. The distributions do show a clear bias to the right hand side of the main peak position, suggesting a steady decrease in pair-distances in going to the total diameter of the $\beta$-particles, not an abrupt decay at a set distance, nor a near perfect Gaussian distribution of distances as might be expected for a perfect sphere [45].

Also shown in Figure 6 are shape reconstructions of one of each normal, T1D, and T2D glycogen $\beta$-particles, constructed using the DAMMIF package [46]. DAMMIF uses simulated annealing to assemble densely packed dummy atoms in such a way that the model yields a scattering pattern that fits experimental data. All reconstructions were done with solvent omitted, no pseudo chains, and with a slow simulated annealing. These reconstructions are only approximations of shape, but normal glycogen $\beta$-particles are clearly elongated in these reconstructions. This may perhaps decay into a polymer-brush-like surface towards the extremity, allowing enzyme access to loose glucose chains. The T1D samples show this same behaviour. An example of a T2D $\beta$-particle shows a more ellipsoidal-like structure. Other shape reconstructions are shown in supporting information, where consistent shapes are found for normal and T1D glycogen $\beta$-particles, but variable shapes for the T2D glycogen $\beta$-particles. The shape of these normal and T1D glycogen $\beta$-particles will result in one axis requiring less torque for angular rotation (a lower inertia tensor). Therefore the elongation of the normal and T1D particles can be quantified by consideration of the inertia tensor. For the tensor across axes $i$ and $k$

$$
I_{j k}=\sum_{i}^{N} m_{i}\left(r_{i}^{2} \delta_{j k}-x_{i j} x_{i k}\right)
$$

where $\delta_{i k}$ is the Kronocker delta, $r$ is the distance to a point, and $m$ is the mass for $N$ coordinates. The moment of inertia tensor, $I$, is symmetric, and as such has orthogonal eigenvectors and real, positive eigenvalues, which can be expressed as 


$$
I_{i k} \hat{\omega}=\lambda \hat{\omega}
$$

where $\hat{\omega}$ is the eigenvector and $\lambda$ the eigenvalue. In this circumstance the eigenvalues represent the principal moments of inertia, and the eigenvectors are the principal axes of rotation. The eigenvalues for each glycogen particle is calculated from their Cartesian coordinates generated via DAMMIF, and compared to see if one vector has a statistically lower eigenvalue for the principal moment of inertia representing it (i.e. does one axis require less torque for rotation?). Results are shown in Table 5 .

Table 5 shows that both T1D and to a greater extent normal $\beta$ particles have one axis that has a lower principal moment of inertia then the other orthogonal axes. From this and Figure 6 it is evident that mammalian glycogen $\beta$-particles, which are capable of forming $\alpha$-particles, are slightly elongated in shape. For more information about the nature of the internal structure of $\beta$-particles, different theories of branching and packing, such as fractal or random branching theory, can now be compared.

Melendez and co-workers [13, 14, 15] have described glycogen $\beta$-particles as biological fractal structures, that are highly optimised to achieve an efficient fuel storage molecule. Melendez and co-workers discuss how glycogen qualities can be regarded as fractal; such as self-similarity between the numbers of external chains in each tier. This description is based on a four parameter optimisation model, which evolves the structure with an optimised density of branches and chains iteratively up to a limit, generally regarded as 12 "tiers" [14]. The optimised parameters, such as the degree of branching, and the number of glucose residues in a chain, have compared well to experiments. However no direct test of the structure has been attempted, to our best knowledge.

On the other hand, Caldwell and Matheson [47] developed a model of random dendritic architecture that uses the characteristic chain distribution and branching pattern of glycogen. They found that the chain distribution of glycogen $\beta$-particles closely resembles their random dendrimeric model [48]. Also based on random architecture is Konkolewicz et al.'s random branching theory (RBT), which has been compared to SAXS profiles of dendrimers of generation zero to eight (see introduction), where the RBT model was shown to capture the conformation of the dendrimers well with a description of randomly branched species of a similar size. Bale and Schmidt [49], Martin and Hurd [50], and also Rieker et al. [51] have shown scattering data for fractal structures that have a clear power-law dependence of $I(q)$. The SAXS profiles of glycogen $\beta$-particles can therefore be used as a test to see if glycogen $\beta$-particles are biological fractal structures or randomly branched polymers.

The scattering from both surface and volume fractals has a simple power-law form [52]

$$
I(q)=I_{0} q^{-\alpha}
$$

where $I_{0}$ and $\alpha$ are constants. Included in Figure 5 is a fit of Equation 4 (black dotted line) to scattering from normal glycogen $\beta$-particles. The best-fit parameters are $I_{0}=1.08251 \times 10^{-3}$, and $\alpha=1.17307$. Figure 5 shows that Equation 4 does not capture the curvature of $I(q)$ with $q$, and therefore the average shape 
of the $\beta$-particles. A simple scaling law such as Equation 4 would not be expected to capture the full profile as $q \rightarrow 0.1 \AA^{-1}$, as this contains contributions from intramolecular correlations. However, since the average shape is not captured by Equation 4, this indicates that glycogen $\beta$-particles are not fractal structures.

For a more rigorous determination of the structural nature of glycogen $\beta$ particles, RBT was compared to the scattering data from normal glycogen $\beta$ particles (see supporting information for calculations) in Figure 7. RBT has five tunable parameters, so the variation in RBT predictions was stress tested with regards to how the predicted $I(q)$ changes with each parameter, shown in Figure 7 with the varied parameters in the supporting information. The theoretical scattering profiles tend to show the correct curvature of $I(q)$ with $q$, but all deviate from experiment as $q \rightarrow 0.1 \AA^{-1}$, which is likely due to a neglect of intramolecular correlations in the RBT model. The model captures the form factor but the deviation at high $q$ could be captured by including a "blob" term, as described by Rathgeber [22, 45]. The Equation 4 fit is also included in Figure 7, which is done so to emphasise the difference between RBT predictions and a power-law scattering. The agreement for scattering profiles between RBT and experiments show that glycogen $\beta$-particles are consistent with a randomly branched structure.

The fitting of RBT additionally allows useful parameters that describe structure to be obtained. For example, RBT fit 1 (see supporting information for parameters) matches the experimental scattering the best, and so from this the number of monomers in a simple chain of certain size from the RBT calculation can be determined. Future work may involve correlating some of these quantities to known data of chain lengths, and then comparing to these SAXS data. Here it has been shown that glycogen $\beta$-particles are consistent with a randomly branched structure, and not a fractal structure.

The use of DAMMIF to model the shape of glycogen particles (Figure 6) involved an algorithm that randomly selects a dummy atom from a configuration and changes its phase (from solvent to particle, or vice versa), and upon defining some criterion, is continued until a configuration is found that fits the experimental data whilst minimising the interfacial area [53]. RBT is also based on an iterative random building process, which starts by randomly assembling simple units together with two main contributions determining the growth probability, being the density of chains for a new unit to add to, and the probability of accommodating a new simple unit in this space. The probability of addition is a convolution of the two functions describing these contributions. Given that RBT can give an estimate of the scattering from a randomly hyperbranched polymer (see supporting information), the RBT scattering prediction can be used to approximate a paircorrelation function via an indirect Fourier transform, and therefore DAMMIF can reconstruct a shape that fits the RBT predictions. This is shown for three RBT calculations in Figure 7.

It is clear that the randomly hyperbranched polymers generated by RBT, that match the experimental SAXS data for $\beta$-particles well, also have an elongated ellipsoidal shape. The RBT predictions compared show little differences between varied parameters, with all predictions consistent with this shape. Included in Table 5 are the calculated eigenvalues for these three RBT shapes, where like with normal and T1D $\beta$-particles, one axis is found to be elongated in comparison to the other two. This demonstrates consistency between the DAMMIF shape predictions for these particle types and RBT polymers. 
It has been shown that SAXS can be performed on mammalian glycogen $\beta$ particles, upon treating the $\alpha$-particles to a gentle hydrolysis procedure. The SAXS profiles provide strong evidence for glycogen $\beta$-particles being randomly branched structures, where RBT modelling fits the experimental data well, and the approximate shape of the $\beta$-particles is anisotropic. No experimental structural evidence has been found that supports glycogen $\beta$-particles being fractal structures, suggesting a revision of the "tier" picture of glycogen is needed. The SAXS data has indicated a more elongated structure for normal mammalian $\beta$-particles, than the typical globular picture. Future work may involve analysing different sized $\alpha$-particles by SAXS to better determine the packing of $\beta$-particles into these large particles. Such work will require purification and isolation of $\alpha$-particles of a particular size in order to remove the polydispersity.

\section{Conclusions}

The structure of liver glycogen $\alpha$ and $\beta$-particles have been investigated by DLS and SAXS, comparing normal and diabetic mice. Not only are there $\alpha$-particles present in T2D livers, but they are present in a much larger loosely bound, aggregate form. This conclusion is based on intense sonication which causes the particles to redisperse into $\alpha$ and $\beta$-particles. An in vitro study of normal glycogen particles in a biological solvent at $37^{\circ} \mathrm{C}$ revealed aggregation occurs over a period of 20 hours. The aggregated glycogen particles found in T2D livers are likely, as a consequence, to have less accessible glucose in times of need. It has been shown that glycogens of different size are metabolised by $\gamma$-amylase at different rates, where the smaller $\beta$-particles are degraded quicker than the larger $\alpha$-particles, and that there are differences in rates between $\alpha$-particles and the larger aggregates in T2D, showing that glucose release from T2D glycogen particles is blunted. This highlights a possible consequence of in vivo aggregate glycogens leading to a poor hypoglycaemic response, as seen in diabetes, but also demonstrates a biologically significant structure-function relationship; the size of glycogen in a tissue determines the rate of glucose release in times of need.

SAXS of glycogen $\beta$-particles have allowed direct comparisons of internal structure to theory, such as fractal and random branching theory, and also allowed the reconstruction of glycogen shape. The data has shown that glycogen $\beta$-particles are not fractal structures, but are consistent with a randomly branched structure. Furthermore, it shows how pair correlations in the electron density across glycogen $\beta$-particles decays, indicate an elongated shape of liver glycogen $\beta$-particles. The anisotropic shape of the $\beta$-particles is very similar to that of a randomly branched polymer.

These findings are summarised in Table 6 . Together, these results show that $\beta$ particles are randomly assembled from oligomeric chains, resulting in an elongated particle. The $\alpha$-particles in turn are assembled from $\beta$-particles. A much weaker force may cause the aggregation of the $\alpha$-particles into larger structures, but these are seen only upon extraction in the T2D samples. It is concluded that this weaker force is likely related to the structure of the glucose chains at the surface of the T2D particles and their time present in vivo, and that the rate of enzyme catalysed release of glucose depends on particle size. This therefore demonstrates a structurefunction relationship for the tissue specific distribution of glycogen particle types. 


\section{Acknowledgements}

The assistance from Dr. Nigel Kirby and Nathan Cowieson at the SAXS beamline is gratefully acknowledged. QAB and XYZ would like to thank the Australian government for APA scholarships, and the Univeristy of Melbourne for receipt of the Albert Shimmins award and the Norma Hilda Schuster scholarship (QAB). This study was partially supported by funding from the Melbourne Materials Institute (QAB, AGW), the Australian Synchrotron (beamline time) (QAB, AGW), the Victorian Life Sciences Computation Initiative (QAB, AGW, Grant No. VRO252), and the Australian Research Council for grant ARCDP110102396 (JMY). 


\section{References}

1. J.H. Ryu, J. Drain, J.H. Kim, S. McGee, A. Gray-Weale, L. Waddington, G.J. Parker, M. Hargreaves, S.H. Yoo, D. Stapleton, Int. J. Biol. Macromol. 45(5), 478 (2009). DOI 10.1016/j.ijbiomac.2009.08.006

2. H.G. Hers, Annu. Rev. Biochem. 45, 167 (1976). DOI 10.1146/annurev.bi.45. 070176.001123

3. P.J. Roach, A.A. Depaoli-Roach, T.D. Hurley, V.S. Tagliabracci, Biochem. J. 441(3), 763 (2012). DOI 10.1042/BJ20111416

4. M. Sakaida, J. Watanabe, S. Kanamura, H. Tokunaga, R. Ogawa, Anat. Rec. 218(3), 267 (1987). DOI 10.1002/ar.1092180307

5. P. Drochmans, Journal of Ultrastructure Research 6(2), 141 (1962). DOI 10.1016/S0022-5320(62)90050-3

6. Q.A. Besford, M.A. Sullivan, L. Zheng, R.G. Gilbert, D. Stapleton, A. GrayWeale, Int. J. Biol. Macromol. 51(5), 887 (2012). DOI 10.1016/j.ijbiomac. 2012.06.037

7. P.O. Powell, M.a. Sullivan, J.J. Sheehy, B.L. Schulz, F.J. Warren, R.G. Gilbert, PLoS One 10(3), e0121337 (2015). DOI 10.1371/journal.pone.0121337

8. M.A. Sullivan, M.J. O' Connor, F. Umana, E. Roura, K. Jack, D.I. Stapleton, R.G. Gilbert, Biomacromolecules 13(11), 3805 (2012). DOI 10.1021/ bm3012727

9. T. Samorajski, J.R. Keefe, J.M. Ordy, Vision Res. 5(1), 639 (1965)

10. G. Bathgate, D. Manners, Biochem. J. 101(1), 3 (1966)

11. D.J. Manners, Carbohydr. Polym. 16(1), 37 (1991). DOI 10.1016/ 0144-8617(91)90071-J

12. M.A. Sullivan, F. Vilaplana, R.A. Cave, D. Stapleton, A.A. Gray-Weale, R.G. Gilbert, Biomacromolecules 11, 1094 (2010)

13. R. Meléndez, E. Meléndez-Hevia, M. Cascante, J. Mol. Evol. 45(4), 446 (1997)

14. E. Meléndez-Hevia, T.G. Waddell, E.D. Shelton, Biochem. J. 295, 477 (1993)

15. R. Meléndez, E. Meléndez-Hevia, E.I. Canela, Biophys. J. 77(3), 1327 (1999). DOI 10.1016/S0006-3495(99)76982-1

16. D. Konkolewicz, R. Gilbert, A. Gray-Weale, Phys. Rev. Lett. 98(23), 1 (2007). DOI 10.1103/PhysRevLett.98.238301

17. D. Konkolewicz, O. Thorn-Seshold, A. Gray-Weale, J. Chem. Phys. 129, 054901 (2008). DOI 10.1063/1.2939242

18. M.P. Hoepfner, C.V.B. Fávero, N. Haji-Akbari, H.S. Fogler, Langmuir 29(28), 8799 (2013). DOI 10.1021/la401406k

19. C.D. Putnam, M. Hammel, G.L. Hura, J.A. Tainer, Q. Rev. Biophys. 40(3), 191 (2007). DOI 10.1017/S0033583507004635

20. E. Goldsmith, S. Sprang, R. Fletterick, J. Mol. Biol. 156(2), 411 (1982)

21. S.K. Filippov, O. Sedlacek, A. Bogomolova, M. Vetrik, D. Jirak, J. Kovar, J. Kucka, S. Bals, S. Turner, P. Stepanek, M. Hruby, Macromol. Biosci. 12(12), 1731 (2012). DOI 10.1002/mabi.201200294

22. D. Konkolewicz, S. Perrier, D. Stapleton, A. Gray-Weale, Journal of Polymer Science Part B: Polymer Physics 49, 1525 (2011). DOI 10.1002/polb.22340

23. J. Berg, J. Tymoczko, L. Stryer, in Biochemistry, 5th edn. (W.H. Freeman, New York, 2002), chap. 12

24. J.E. Gerich, Bailliere's Clinical Endocrinology and Metabolism 7(3), 551 (1993) 
25. S.E. Leney, J.M. Tavaré, The Journal of Endocrinology 203(1), 1 (2009). DOI 10.1677/JOE-09-0037

26. B.B. Kahn, J.S. Flier, J. Clin. Invest. 106(4), 473 (2000)

27. A.R. Saltiel, C.R. Kahn, Nature 414, 799 (2001)

28. C. Postic, R. Dentin, J. Girard, Diabetes and Metabolism 30(5), 398 (2004)

29. H.G. Hers, T.D. Barsy, B. Lederer, L. Hue, F. Van Hoof, Birth Defects Original Article Series 12(6), 145 (1974)

30. F. Nitschke, P. Wang, P. Schmieder, J.M. Girard, D.E. Awrey, T. Wang, J. Israelian, X. Zhao, J. Turnbull, M. Heydenreich, E. Kleinpeter, M. Steup, B.A. Minassian, Cell Metab. 17(5), 756 (2013). DOI 10.1016/j.cmet.2013.04.006

31. P.J. Roach, Molecular Aspects of Medicine (2015). DOI 10.1016/j.mam.2015. 08.003

32. G. Jiang, B.B. Zhang, American Journal of Endocrinology and Metabolism 284(4), 671 (2003). DOI 10.1152/ajpendo.00492.2002

33. P.E. Cryer, N. Engl. J. Med. 369(4), 362 (2013). DOI 10.1056/ NEJMra1215228

34. N.N. Zammitt, B.M. Frier, Diabetes Care 88(4), 1107 (2005). DOI 10.1016/ j.mcna.2004.04.003

35. M. Krssak, A. Brehm, E. Bernroider, C. Anderwald, P. Nowotny, C.D. Man, C. Cobelli, G.W. Cline, G.I. Shulman, W. Waldha, M. Roden, Diabetes 53, 3048 (2004)

36. P.A. Mayes, D.A. Bender, in Harper's Biochemistry, 23rd edn. (Appleton \&amp; Lange, Norwalk CT, 1993), chap. 18, pp. 145-152

37. M.A. Sullivan, J. Li, C. Li, F. Vilaplana, D. Stapleton, A.A. Gray-Weale, S. Bowen, L. Zheng, R.G. Gilbert, Biomacromolecules 12(6), 1983 (2011). DOI $10.1021 / \mathrm{bm} 2006054$

38. X.Y. Zeng, Y.P. Wang, J. Cantley, T.J. Iseli, J.C. Molero, B.D. Hegarty, E.W. Kraegen, Y. Ye, J.M. Ye, PLoS One 7(7), e42115 (2012). DOI 10.1371/journal. pone. 0042115

39. M. Kaszuba, D. McKnight, M.T. Connah, F.K. McNeil-Watson, U. Nobbmann, J. Nanopart. Res. 10(5), 823 (2007). DOI 10.1007/s11051-007-9317-4

40. B. Deng, M.a. Sullivan, J. Li, X. Tan, C. Zhu, B.L. Schulz, R.G. Gilbert, Glycoconjugate J. 32, 113 (2015). DOI 10.1007/s10719-015-9578-6

41. N. Arjmandi, W. Van Roy, L. Lagae, G. Borghs, Anal. Chem. 84(20), 8490 (2012). DOI 10.1021/ac300705z

42. V.S. Tagliabracci, C. Heiss, C. Karthik, C.J. Contreras, J. Glushka, M. Ishihara, P. Azadi, T.D. Hurley, A.a. Depaoli-Roach, P.J. Roach, Cell Metab. 13(3), 274 (2011). DOI 10.1016/j.cmet.2011.01.017

43. I. Magnusson, D.L. Rothman, L.D. Katz, R.G. Shulman, G.I. Shulman, J. Clin. Invest. 90(4), 1323 (1992). DOI 10.1172/JCI115997

44. J.N. Clore, W.G. Blackard, Diabetes 43, 256 (1994)

45. S. Rathgeber, M. Monkenbusch, M. Kreitschmann, V. Urban, A. Brulet, J. Chem. Phys. 117(8), 4047 (2002). DOI 10.1063/1.1493771

46. D. Franke, D. Svergun, J. Appl. Crystallogr. 42, 342 (2009)

47. R. Caldwell, N. Matheson, Carbohydr. Polym. 54(2), 201 (2003). DOI 10. 1016/S0144-8617(03)00161-9

48. N.K. Matheson, R.A. Caldwell, Carbohydr. Polym. 40, 191 (1999)

49. H.D. Bale, P.W. Schmidt, Phys. Rev. Lett. 53(6), 596 (1984) 
50. J.E. Martin, A.J. Hurd, J. Appl. Crystallogr. 20(2), 61 (1987). DOI 10.1107/ S0021889887087107

51. T.P. Rieker, M. Hindermann-Bischoff, F. Ehrburger-Dolle, Langmuir 16(13), 5588 (2000). DOI 10.1021/la991636a

52. M.H. Reich, I.K. Snook, H.K. Wagenfeld, Fuel 71, 669 (1992)

53. D.I. Svergun, Biophys. J. 76, 2879 (1999). DOI 10.1016/S0006-3495(99) 77443-6 


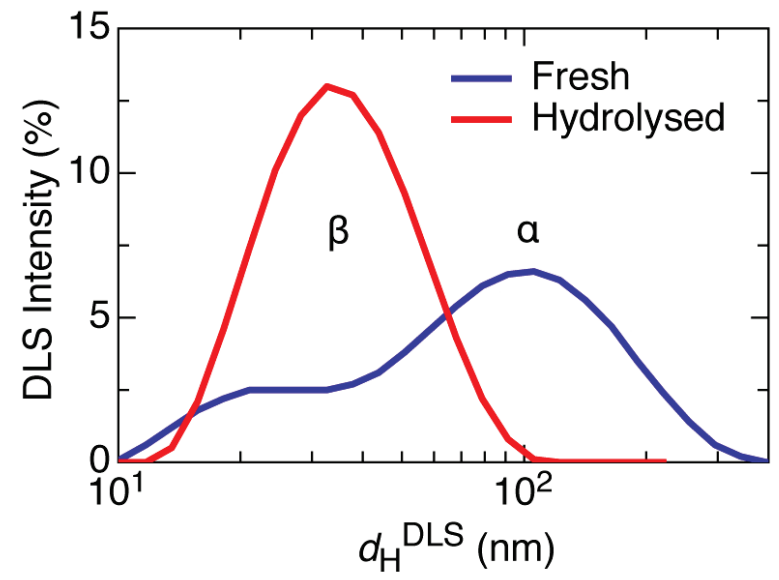

Fig. 1 DLS confirming the hydrolysis of normal mouse liver glycogen $\alpha$-particles into particles of $\beta$-particle size. This measurement is taken after the hydrolysis treatment, as described in methods, prior to SAXS analysis.

\begin{tabular}{cccc}
\hline \hline & normal & T1D & T2D \\
\hline BGL $(\mathrm{mM})$ & $9.4 \pm 0.4$ & $13.6 \pm 0.6^{*}$ & $18.9 \pm 0.8^{* \dagger}$ \\
\hline \hline
\end{tabular}

Table 1 The fasting blood glucose level (BGL) of the animals sacrificed( * $\mathrm{p}<0.01$ vs normal, $\dagger \mathrm{p}<0.01$ vs T1D $(\mathrm{n}=5-7 /$ group $))$

\begin{tabular}{lcc}
\hline \hline Model & $\zeta$-potential $/ \mathrm{mV}$ & $\sigma / \mathrm{mV}$ \\
\hline Control & -23.6 & 0.351 \\
T2D & -21.3 & 0.702 \\
T1D & -28.6 & 1.49 \\
\hline \hline
\end{tabular}

Table $2 \zeta$-potentials of mouse liver glycogen particles from normal, T1D, and T2D animals. Measurements were performed in deionised water.

\begin{tabular}{lccccc}
\hline \hline Glycogen source & Particle type & $N$ & $\tau_{r} /$ min $^{-1}$ & $S E_{\text {exp }} /$ min $^{-1}$ & $S E_{f i t} /$ min $^{-1}$ \\
\hline T2D liver & aggregate & 7 & 21.97 & $3.24 x 10^{-3}$ & 0.525 \\
T1D liver & $\alpha$ & 3 & 18.53 & $2.20 x 10^{-4}$ & 0.475 \\
Rabbit liver & $\beta$ & & 10.50 & - & 0.140 \\
Oyster & $\beta$ & & 8.89 & - & 0.151 \\
\hline \hline
\end{tabular}

Table 3 The relaxation times for the enzyme degradation $\left(\tau_{r}\right)$ by $\gamma$-amylase for various glycogen types, along with the standard error from experiment $\left(S E_{\text {exp }}\right)$ and for the fits $\left(S E_{f i t}\right)$. See supporting information for fitted data. 

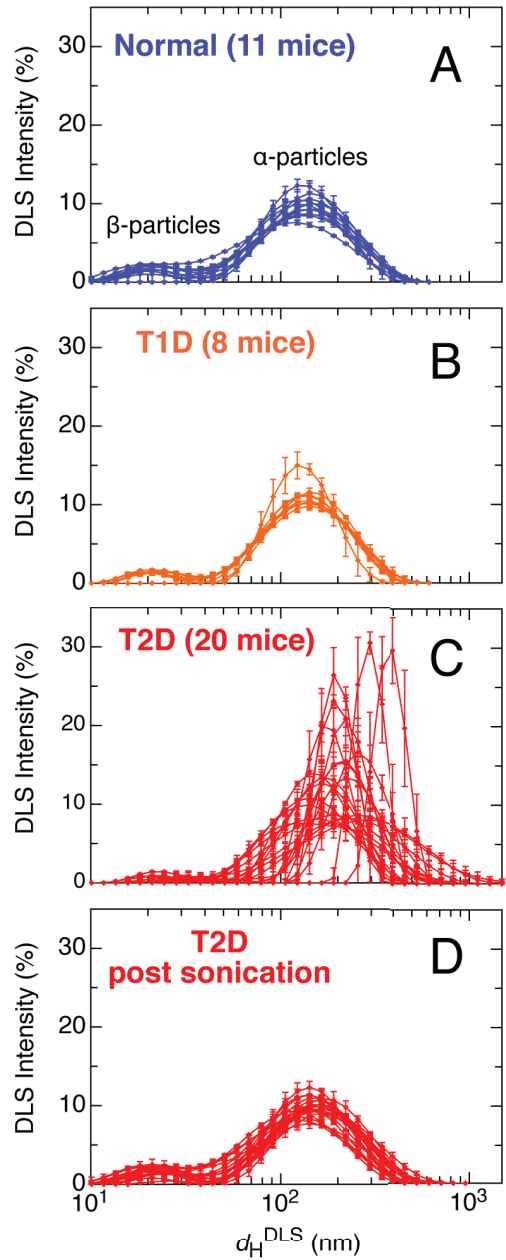

Fig. 2 DLS of mouse liver glycogen $\alpha$ and $\beta$-particles from normal (A), type 1 diabetic (T1D) (B), type 2 diabetic (T2D) (C), and T2D after intense sonication (D), mice. All samples were analysed at $298 \mathrm{~K}$. Each distribution is the average of 3 runs, each consisting of 15 independent measurements, with the standard deviation between measurements shown as error bars.

\begin{tabular}{lccccc}
\hline \hline Model & $\mathrm{n}$ & $r_{g}^{S A X S}$ & $\sigma_{s e}^{S A X S}$ & $r_{h}^{D L S}$ & $\sigma_{s e}^{D L S}$ \\
\hline normal & 5 & 18.38 & 0.88 & 21.94 & 3.89 \\
T2D & 5 & 22.78 & 1.06 & - & - \\
T1D & 3 & 18.35 & 0.19 & - & - \\
\hline
\end{tabular}

Table 4 Radii of gyration as calculated from a Guinier fit to the scattering profiles, where $r_{g}^{S \bar{A} X S}$ is the average radii of gyration from SAXS, $\sigma_{s e}^{S A X S}$ the standard error of the average, $r_{\text {DLS }}^{D L}$ is the hydrodynamic radius from DLS, along with its standard error $\sigma_{s e}^{D L S}$. The units of all radii and errors are nm. No DLS data for the hydrolysed diameters of the diabetic particles was recorded. 


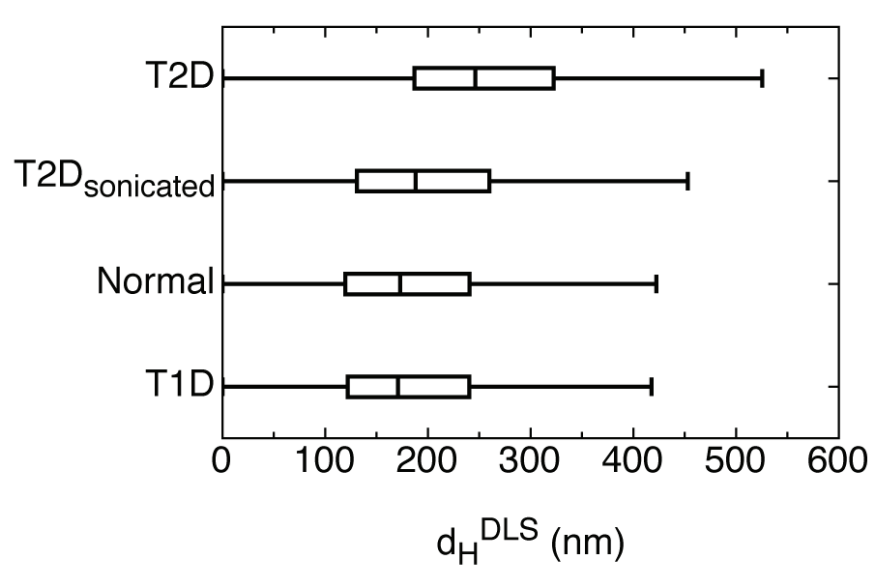

Fig. 3 Boxplot showing the $Q 1, Q 2$ (median), and $Q 3$ for all samples. Statistics calculated from DLS intensity distributions in Figure 2.

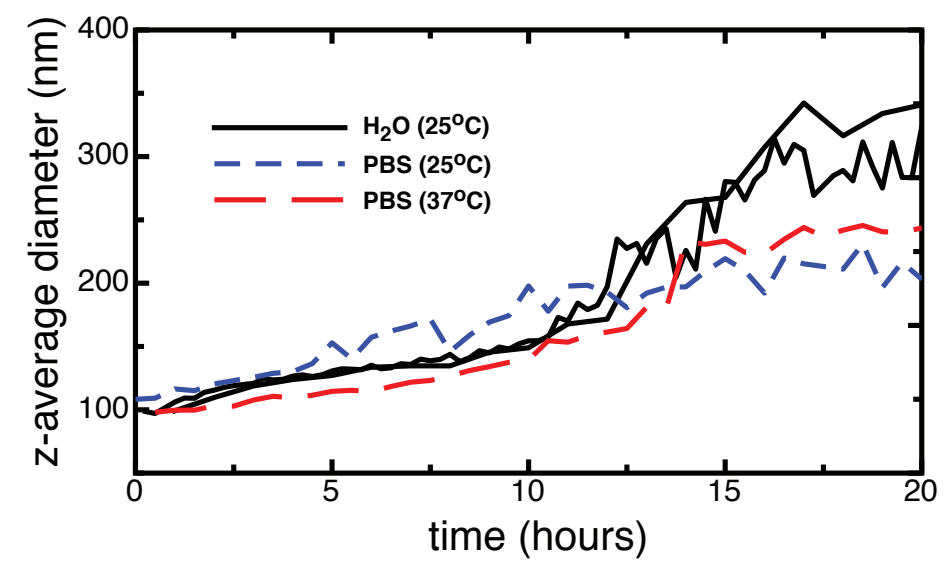

Fig. 4 The z-average diameter of normal glycogen particles over time in $\mathrm{H}_{2} \mathrm{O}$ at $25^{\circ} \mathrm{C}$ (two samples, solid black lines), in PBS buffer at $25^{\circ} \mathrm{C}$ (blue dotted line), and in PBS buffer at $37^{\circ} \mathrm{C}$ (blue dashed lines).

\begin{tabular}{lccc}
\hline Particle type & $\lambda_{i}$ & $\lambda_{j}$ & $\lambda_{k}$ \\
\hline normal $\beta$ & $3.02 \pm 0.151$ & $3.09 \pm 0.263$ & $0.714 \pm 0.167$ \\
T1D $\beta$ & $4.69 \pm 0.740$ & $3.66 \pm 0.515$ & $1.50 \pm 0.716$ \\
RBT 1 & 1.52 & 1.18 & 0.599 \\
RBT 3 & 1.97 & 1.69 & 0.589 \\
RBT 6 & 1.59 & 1.40 & 0.491 \\
\hline
\end{tabular}

Table 5 A comparison of eigenvalues $(\lambda)$ for the axes $i, j$, and $k$, for normal and T1D $\beta$ particles, and RBT polymers. The units of $\lambda$ are $10^{7} m \AA^{2}$, where $m$ is taken as unity. The errors are standard errors across 5 normal and 3 T1D animals. 


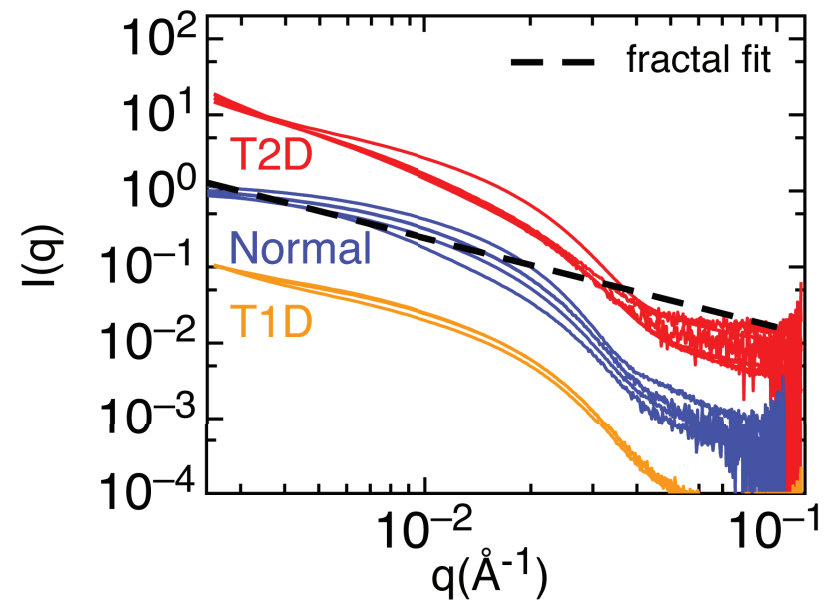

Fig. 5 Solvent subtracted SAXS intensity profiles for normal ( 5 animals), T1D (3 animals), and T2D ( 5 animals) mouse liver glycogen $\beta$-particles. The curves between different animal types are offset for clarity (T2D x10 $10^{1}$, normal $\times 10^{1}$, and T1D $\times 10^{-1}$. Also included is a simple power law form for fractal scattering calculated from Equation 4 (black dotted line).

\begin{tabular}{|c|c|}
\hline & Finding \\
\hline glycogen & different particle sizes release glucose at different rates \\
liver glycogen & particles aggregate with time \\
T2D liver glycogen & initially present as large aggregates with blunted glucose release \\
liver glycogen & randomly branched polymers, not fractal \\
\hline
\end{tabular}

Table 6 A summary of the main findings in this work 

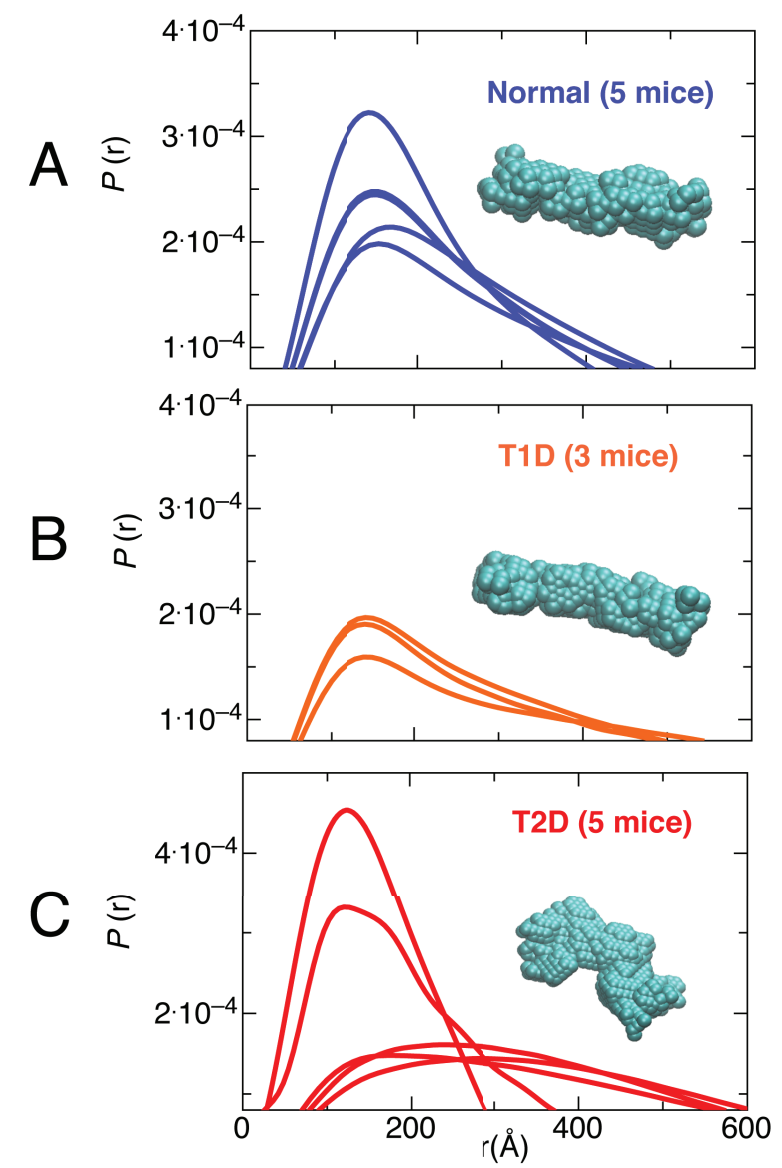

Fig. 6 Normalised pair-distribution functions $(P(r))$ of glycogen $\beta$-particles from normal (5 animals), T1D (3 animals) and T2D (5 animals) mouse livers, as obtained from SAXS data. Included in each plot are shape reconstructions from the scattering data of each type of glycogen $\beta$-particle (see text). 


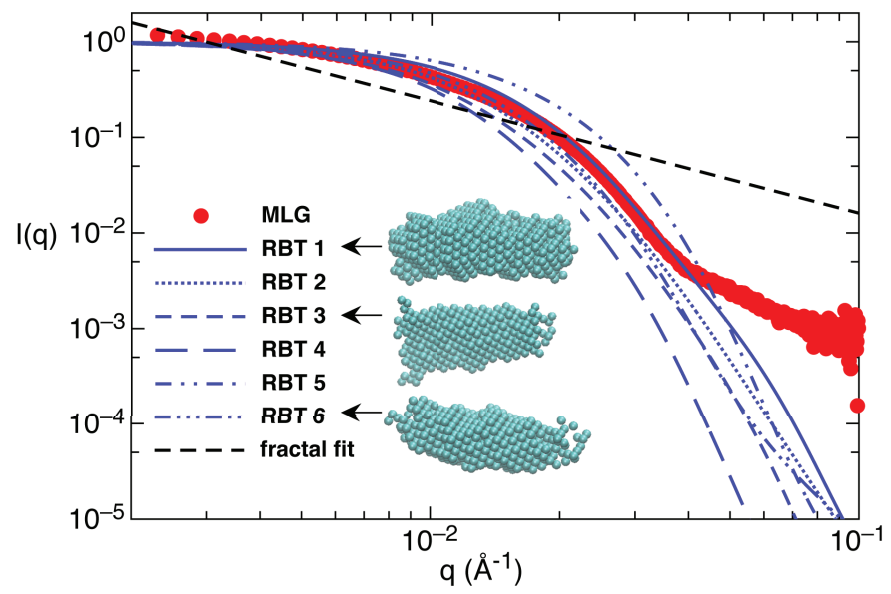

Fig. 7 Stress testing of RBT models against experimental SAXS measurements for normal mouse liver glycogen (MLG) $\beta$-particles. Parameters are shown in supporting information. Also shown is the expected scattering from a fractal object (Equation 4), along with DAMMIF shape reconstructions of three RBT profiles. 


\section{University Library}

\section{- M M N E R VA A gateway to Melbourne's research publications}

Minerva Access is the Institutional Repository of The University of Melbourne

Author/s:

Besford, QA;Zeng, X-Y;Ye, J-M;Gray-Weale, A

Title:

Liver glycogen in type 2 diabetic mice is randomly branched as enlarged aggregates with blunted glucose release

Date:

2016-02-01

\section{Citation:}

Besford, Q. A., Zeng, X. -Y., Ye, J. -M. \& Gray-Weale, A. (2016). Liver glycogen in type 2 diabetic mice is randomly branched as enlarged aggregates with blunted glucose release. GLYCOCONJUGATE JOURNAL, 33 (1), pp.41-51. https://doi.org/10.1007/ s10719-015-9631-5.

Persistent Link:

http://hdl.handle.net/11343/282927 